\title{
ENHANCEMENT OF THE POSITRON INTENSITY BY A TUNGSTEN SINGLE-CRYSTAL TARGET AT THE KEKB INJECTOR LINAC
}

\author{
T. Suwada*, M. Satoh, T. Sugimura, T. Kamitani, K. Furukawa, K. Umemori, and H. Okuno, \\ KEK, Tsukuba, Japan \\ T. Haruna, R. Hamatsu, T. Sumiyoshi, Tokyo Metropolitan University, Hachioji, Japan \\ K.Yoshida, SAGA Light Source, Tosu, Japan \\ A.P. Potylitsin, I.S. Tropin, Tomsk Polytechnic University, Tomsk, Russia \\ R. Chehab, IPNL, IN2P3-CNRS, Universite Claude Bernald 1, France
}

\begin{abstract}
A new tungsten single-crystal target has been successfully employed at the positron source of the KEKB injector linac. The crystal thickness was determined to be $10.5 \mathrm{~mm}$ based on the previous systematic measurements of the positron-production efficiency. The crystal axis, $<111>$, was precisely aligned to the direction of the $4-\mathrm{GeV}$ primary electron beam. The positron yield increased by $\sim 25 \%$ compared to that for a conventional tungsten plate with a thickness of $14 \mathrm{~mm}$. On the contrary, the heat load on the crystal target decreased by $\sim 20 \%$. No degradation of the positronproduction efficiency was observed since the crystal target was installed in September 2006, and it has been stably operating at the KEK B-factory.
\end{abstract}

\section{INTRODUCTION}

In order to achieve high luminosities in high-energy $e^{+} e^{-}$collider experiments, positron sources must be reinforced, especially towards next generation of Bfactories and $e^{+} e^{-}$linear colliders. In a conventional positron source, positrons are produced by high-energy electrons hitting a heavy-metal target, and lowmomentum positrons are captured and accelerated in the succeeding positron capture section. The maximum positron-production efficiencies are obtained with an optimized target thickness of $4-5 X_{0}$ and with a typical momentum acceptance of $5-25 \mathrm{MeV} / \mathrm{c}$ for $4-8 \mathrm{GeV}$ incident electrons. In this case, a possible scheme to increase the positron intensity is to increase the incident electron intensity and energy. However, the allowable heat load on the target limits the beam power of the incident electrons.

One promising approach to improve the positronproduction efficiency is to use a crystal-assisted positron source first proposed by Chehab, et al. [1] in 1989. When high-energy electrons hit a single crystal in the direction of the crystal axis, intense low-energy photons are produced due to channeling radiation and coherent bremsstrahlung. These photons create $e^{+} e^{-}$pairs in the same crystal target. Thus, it is expected that the crystal target could increase the obtainable maximum positrons compared with a conventional heavy-metal target.

Intensive theoretical investigations [2,3] have indicated that the optimum thickness is less for the crystal target

*E-mail address: tsuyoshi.suwada@kek.jp. and that the energy deposition in the crystal target is considerably reduced compared with that of a conventional target. Also, they have shown that the crystal effect is much larger at higher incident electron energies.

Encouraged by these stimulating results, we proceeded to systematic studies on the positron-production efficiencies with tungsten crystals having various thicknesses using 4- and $8-\mathrm{GeV}$ electron beams $[4,5]$. Based on these results, we optimized the thickness of the tungsten crystal at a $4-\mathrm{GeV}$ primary electron beam. After developing a crystal-axis alignment technique, we installed a tungsten crystal target at the positron source of the KEKB injector linac.

\section{THE KEKB POSITRON SOURCE}

The KEKB positron source comprises a positronproduction target and a positron capture section. The target is composed of a conventional tungsten plate, and the capture section is employed for a so-called quarter wave transformer. The design and its performance are described elsewhere [6]. The positrons are generated by the 4-GeV primary electron beam impinging on the tungsten target. The average beam power is $2 \mathrm{~kW}$ at a maximum repetition rate of $50 \mathrm{~Hz}$. The typical transverse beam size is $0.7 \mathrm{~mm}$ (rms) in radius, and the typical horizontal and vertical normalized emittances are 660 $\mathrm{mm} \cdot \mathrm{mrad}$ and $360 \mathrm{~mm} \cdot \mathrm{mrad}(\mathrm{rms})$ on average at the target. The horizontal (vertical) angular spread at the target is estimated to be $0.2(0.1) \mathrm{mrad}(\mathrm{rms})$. These angular spreads need to be controlled within the critical angle for axial channeling, which is $0.61 \mathrm{mrad}$ at $4 \mathrm{GeV}$ in a tungsten crystal.

The previously used 14 -mm-thick $\left(4 X_{0}\right)$ conventional tungsten plate was replaced by a 10.5 -mm-thick tungsten crystal in September 2006 without any significant modification of the accelerator layout.

\section{TUNGSTEN CRYSTAL TARGET}

\section{Optimization of the Target Thickness}

For practical applications of the crystal target, we systematically studied the positron-production efficiencies as a function of the crystal thickness at an incident electron energy of $4 \mathrm{GeV}$ at the KEKB injector linac. The positrons produced in the forward direction were measured by a magnetic spectrometer with an 
angular acceptance of about $1 \mathrm{msr}$ and a typical momentum acceptance of $2.4 \%$ (at the momentum of 20 $\mathrm{MeV} / c$ ) in the momentum range of 5-20 MeV/c. Tungsten crystal targets with various thicknesses were tested and the optimum thickness was investigated with an incident electron energy of $4 \mathrm{GeV}$. Figure 1 shows the results of the positron-production efficiencies measured at a momentum of $20 \mathrm{MeV} / c$.

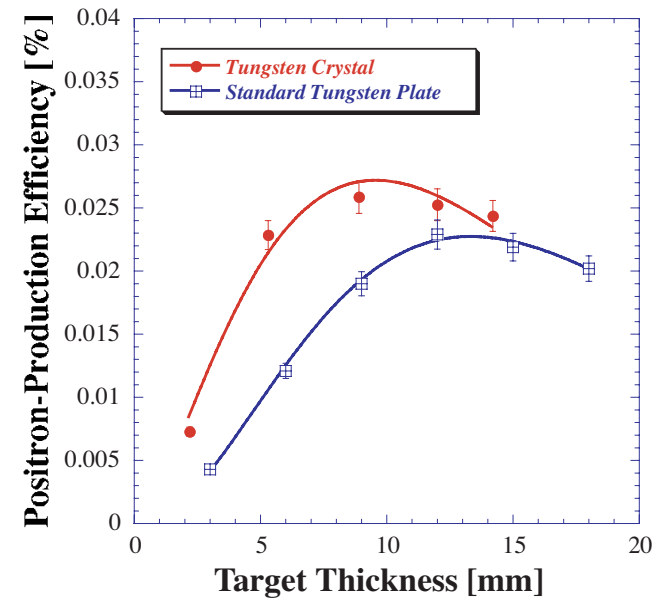

Figure 1: Positron-production efficiencies measured for the tungsten crystal as a function of the crystal thickness (see [5]). The solid curves through the data are gammafunction fits of the data.

The optimum thickness giving the maximum positronproduction efficiency was obtained to be about $10 \mathrm{~mm}$. As a comparison, it was about $14 \mathrm{~mm}$ for the conventional tungsten plate. From these data, it was concluded that the maximum positron yield for the 10mm-thick tungsten crystal target was about $26 \%$ larger than that for the conventional tungsten plate at a momentum of $20 \mathrm{MeV} / c$. The peak width of the rocking curve was also obtained to be 40 mrad in FWHM (see [5]). The relatively large peak width helps us to relax the tolerance of the target axis alignment with respect to the direction of the primary electron beam.

\section{Target Fabrication}

Figure 2 (a) shows a mechanical drawing of the target assembly with a tungsten crystal target. The crystal target with a thickness of $10.5 \mathrm{~mm}$ and a cross section of $5 \mathrm{~mm}$ $\times 5 \mathrm{~mm}$ is fixed at the center of a cylindrical copper body for water cooling with a hot isostatic pressing technique. Figure 2 (b) also shows the target assembly installed in a vacuum chamber. The heat at the target is conducted through a cooling water channel wound around a copper body of $50-\mathrm{mm}$ diameter. Two thermocouples are mounted near the tungsten crystal in order to measure the temperature rise. The target assembly linked with a mechanical linear actuator is mounted in a vacuum chamber. It is inserted into the beam line by the linear actuator for an injection request of the positron beam.

Since there was no room to install a goniometer for controlling the crystal axis with respect to the incident electron beam, the target assembly was carefully fabricated so that the central axis of the cylindrical copper body corresponded exactly to the crystal axis, $<111>$. In order to precisely align these two axes, the relative inclination angles between them were obtained by setting the copper body on a positioning jig and by measuring the crystal axis with an $X$-ray Bragg reflection method. Based on the results, the copper body was mechanically machined so as to correct for any misalignment.

(a)

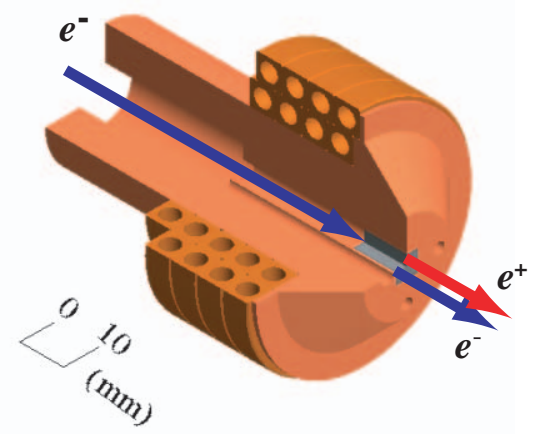

(b)

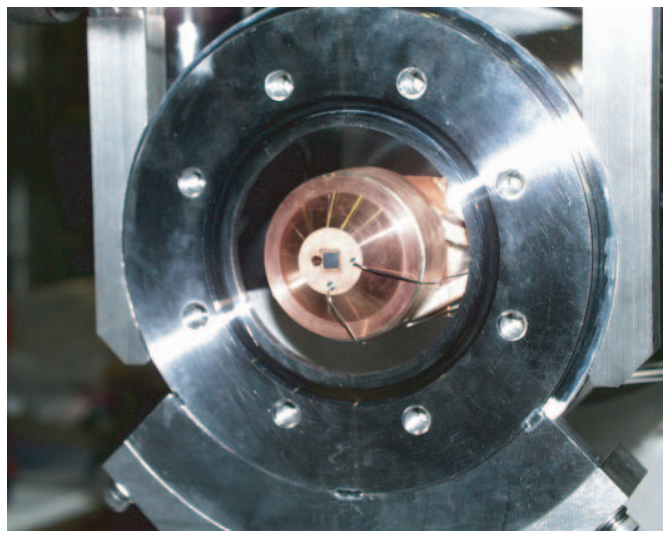

Figure 2: (a) Mechanical drawing of the target assembly. Primary electrons (blue arrow) impinge the tungsten crystal target and are converted to electrons (blue arrow) and positrons (red arrow). (b) Target assembly installed in a vacuum chamber seen from downstream.

\section{PERFORMANCE OF THE CRYSTAL TARGET}

\section{Positron-production Efficiency}

Beam tests were carried out by adjusting the incident angles of the primary electron beam at the crystal target with two sets (horizontal and vertical) of upstream steering magnets. The charges of the primary electron beam was $7.5 \mathrm{nC} /$ bunch on average during the beam tests.

After optimization of the two sets of steering magnets, both the positron and primary electron charges were simultaneously measured with the upstream and downstream beam-position monitors. Figure 3 shows the distributions of the positron-production efficiencies $\left(\mathrm{Ne}^{+} / \mathrm{Ne}^{-}\right)$of the 1st bunch measured for each beam pulse, where $\mathrm{Ne}^{+}$is the number of positrons captured in the positron capture section and $N_{e}^{-}$is the number of the primary electrons. 


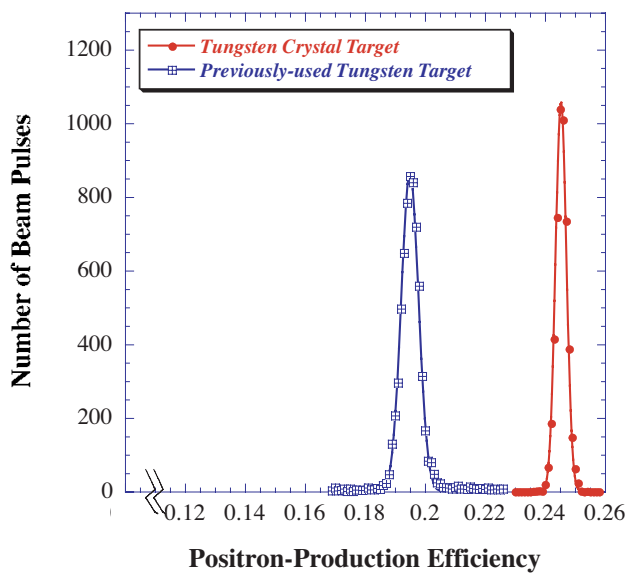

Figure 3: Positron-production efficiencies of the 1st bunch measured for each beam pulse. For the sake of comparison, the data of the previous tungsten plate are plotted. The solid lines are Gaussian-function fits of the data.

For the sake of comparison, the data with the previously used conventional tungsten plate obtained in June 2006 are also shown in this figure. The results show that the positron-production efficiencies of the first (second) bunch are $0.25 \pm 0.01(0.26 \pm 0.01)$ and $0.2 \pm 0.01$ $(0.2 \pm 0.01)$ on average for the tungsten crystal target and the previously used tungsten plate, respectively. The increase in the positrons for the 1st (2nd) bunch from the crystal tungsten is $25 \pm 2 \%(28 \pm 2 \%)$ on average, where the errors indicate one standard-deviation uncertainties. The positron-production efficiencies of both bunches are consistent with each other within the experimental errors. These results are quantitatively in agreement with our previous results obtained by experimental studies described before.

\section{Heat Load on the Crystal Tungsten}

The temperature rise of the crystal target was also measured with two thermocouples mounted inside the copper body. The temperature rise is defined by the average temperature of the two thermocouples. The temperature rise was $\Delta T \approx 13.2{ }^{\circ} \mathrm{C}$ at a beam repetition rate of $50 \mathrm{~Hz}$ in one-bunch operation with a bunch charge of $7.8 \mathrm{nC}$, on average. Figure 4 shows the variations in the temperature rise of the crystal target normalized by the charges of the primary electron beam as a function of the beam repetition rate along with the data of the previously used tungsten plate.

These results confirm that the heat load on the crystal target clearly decreased by about $20 \%$ compared with that of the previously used tungsten plate. The energy deposits on the crystal and conventional targets has been investigated theoretically by Artru, et al. [3] at an incident electron energy of 2-10 GeV. Based on their results, the total energy deposit on a 10-mm-thick crystal is estimated to be $\sim 20 \%$ lower than that of a 14-mm-thick conventional tungsten at an incident electron energy of 4
$\mathrm{GeV}$. The present result is consistent with their theoretical estimation.

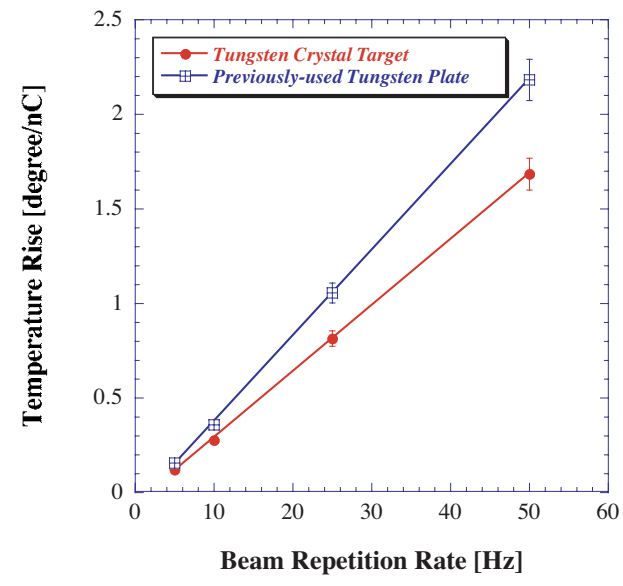

Figure 4: Variations of the temperature rise of the tungsten crystal target as a function of the beam repetition rate in one-bunch operation.

\section{SUMMARY}

We have successfully applied a new tungsten crystal target for generating an intense positron beam at the positron source of the KEKB injector linac. The positron intensity increased by $\sim 25 \%$ compared with that from the previously used conventional tungsten plate, and on the contrary, the heat load on the crystal target decreased by $\sim 20 \%$. It has boosted the positron intensity to its maximum since the beginning of the KEKB operation in 1999. This is the first application of a crystal target to high-energy electron/positron linacs. Useful information concerning radiation damage and stability of the crystal target will be obtained through the long-term KEKB operation.

\section{ACKNOWLEDGEMENT}

This work was supported by the collaborative R\&D program at KEK, by the Grant-in-Aid of Ministry of Education, Culture, Sports, Science and Technology (MEXT) of Japan (Grant No.17540377), and by the grant of Ministry of Education and Science of the Russian Federation "Advancement of the Scientific Potential of High Education" (Grant No.2.1.1.889).

\section{REFERENCES}

[1] R. Chehab, et al., PAC'89, Chicago, IL, U.S.A., 1989, p. 283.

[2] V.N. Baier and V.M. Strakhovenko, Phys. Rev. ST Accel. Beams 5, 121001 (2002).

[3] X. Artru, et al., Phys. Rev. ST Accel. Beams 6, 091003 (2003).

[4] T. Suwada, et al., Phys. Rev. E 67 (2003) 016502.

[5] T. Suwada, et al., LINAC'06, Knoxville Convention Center, TN, U.S.A., 2006, p. 797.

[6] A. Enomoto, et al., EPAC'92, vol.1, Berlin, Germany, 1992, p. 524. 